\title{
Aspergillus endocarditis presenting as acute right lower limb pain
}

\author{
Ghosh $\mathrm{P}^{1}$, Saha $\mathrm{K}^{2}$ \\ ${ }^{l}$ Department of Pathology, NEON Lab, Kolkata, India \\ ${ }^{2}$ Department of Pathology, Institute of Post Graduate Medical Education and Research, Kolkata, India
}

\section{Keywords:}

Aspergillus;

Endocarditis;

Embolism

\begin{abstract}
Aspergillus endocarditis is an 2nd most common cause of endocarditis having poor prognosis. We report a rare case of fungal endocarditis following cardiac valve replacement presenting as acute pain in the right lower limb. Embolism and endocarditis were primarily detected by CT angiography and echocardiography respectively. Diagnosis was established by histopathology and culture of the emboli and was confirmed later by isolation of the Aspergillus species from the resected valve tissue.
\end{abstract}

\section{INTRODUCTION}

Aspergillus endocarditis is the $2^{\text {nd }}$ most common form of fungal endocarditis after that caused by Candida species and occurs in prosthetic valve recipients and in native cardiac valves in intravenous drug users and patients with indwelling central venous catheters. Clinically, these patients present with fever and embolic complications. ${ }^{1}$ Aspergillus species can give rise to a spectrum of disorders, some reflecting hypersensitivity responses to the organisms, and others as the consequences of invasive infection. Distinguishing among these presentations is critical for proper management. ${ }^{2}$ It is therefore of utmost importance for a clinician to take the invaluable assistance from radiologist, surgical pathologist and microbiologist to arrive at the final diagnosis. ${ }^{3}$ Here, we report a case of postoperative Aspergillus endocarditis presenting with acute pain in right lower limb.

\section{Correspondence:}

\section{Dr. Kaushik Saha, MD}

Institute of Post Graduate Medical Education and Research, Kolkata,India Email:drkaushik.saha@gmail.com

\section{CASE REPORT}

A 62 year-old diabetic and hypertensive female patient was admitted to an emergency department for an acute onset right lower limb pain since last 5 days. Patient was on medication for diabetes and hypertension. She underwent mitral valve replacement (MVR) seven months back. On examination she was febrile $\left(101^{\circ} \mathrm{F}\right)$, blood pressure was $160 / 88 \mathrm{~mm}$ Hg. She had a pale and cold right lower limb with pulseless right femoral artery.

Her investigations at the time of admission revealed normal levels of hemoglobin, blood glucose, urea and creatinine. Her total WBC count was $22,800 / \mathrm{mm} 3$ with polymorphonuclear cells $78 \%$, lymphocytes of $12 \%$, eosinophils of $8 \%$ and monocytes of $2 \%$, platelet count was $102,000 / \mathrm{mm}^{3}$, normal CRP $(2 \mathrm{mg} / \mathrm{dl})$ and ESR $(130 \mathrm{~mm}$ at the end of 1 hour).

On the first post-admission day CT angiography (CTA) 
of right lower limb demonstrated multiple emboli in the right femoral artery. Embolectomy was performed. Transthoracic echocardiography revealed moderate to severe aortic insufficiency with large mobile vegetations on the aortic valve. After one week following embolectomy aortic valve replacement was done.

Embolectomy specimen was received for histopathology and for culture. Grossly, the embolectomy specimens were elongated, blackish brown, three in number with each measuring approximately 2 to $3 \mathrm{~cm}$ in length. The specimens were routinely processed and stained with hematoxylin and eosin (HE).

The histopathological sections demonstrated organized vascular embolus infiltrated mostly by neutrophils. In addition we got the areas of alternating staining intensity that were evident (fig.1A), giving the impression of alternating zones of fungal growth. Plenty of fungal septate acute angle dichotomous branching hyphae of Aspergillus species were identified under higher power of microscope (fig.1B). Periodic acid-Schiff (PAS) stain was done for better demonstration of fungal hyphae (fig.2). A $10 \% \mathrm{KOH}$ preparation also revealed similar kind of fungal hyphae. Culture in Sabouraud dextrose agar (SDA) medium subsequently produced heavy growth of Aspergillus species.

\section{DISUCSSION}

True Aspergillus valvular endocarditis is extremely rare and mainly occurs after cardiac surgery on prosthetic valves. $^{4} \quad$ Fungal endocarditis has been found to affect aortic valve prosthesis most commonly and to occur soon after valve surgery. ${ }^{5}$ Verghese $\mathrm{S}$ et al reported two cases of postoperative Aspergillus endocarditis in immunocompetent patients presenting as femoral artery embolism following cardiovascular surgery. ${ }^{6}$ Diagnosis was established by culture of the emboli and was confirmed later by isolation of the same Aspergillus species from the resected valve tissue. Noordally SO et al reported a case of postoperative Aspergillus niger aortitis in an immunocompromised patient presenting as femoral artery embolism. ${ }^{7}$ Rana $\mathrm{M}$ et al observed a very interesting case of embolic Aspergillus endophthalmitis from the hematogenous spread of Aspergillus infection from aortic root eight months following composite aortic root replacement in a young immunocompetent person for severe aortic regurgitation and dilated ascending aorta. ${ }^{8}$

In the present case Aspergilli could have gained entry from the environment during surgery. The use of echocardiography and CTA suggested the provisional diagnosis of embolism following endocarditis but the final diagnosis was ultimately established by histopathology and confirmed by culture and isolation of fungus from the specimens of embolectomy and infected cardiac valve.

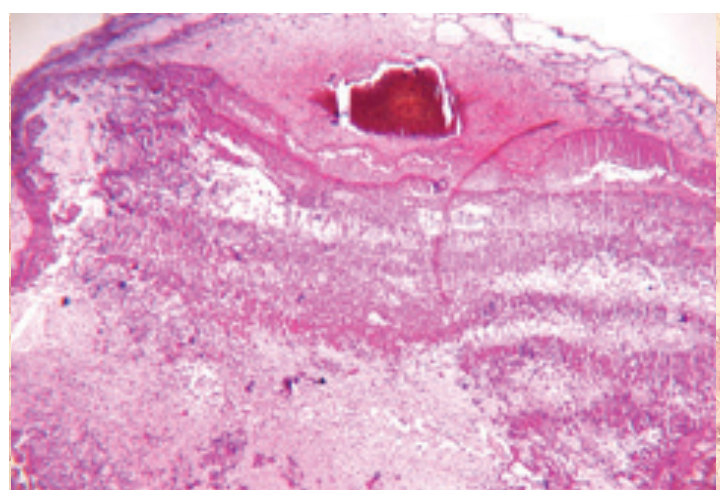

Figure 1A: Areas of alternating staining intensity indicating alternating zones of fungal growth. (HE stain, X40).

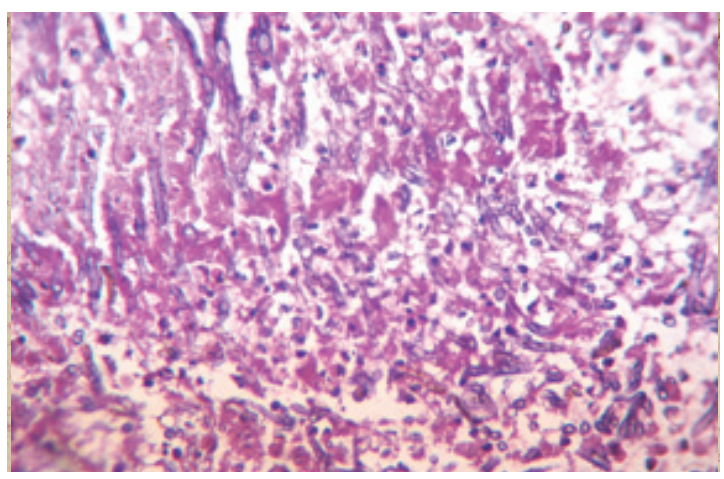

Figure 1B: Plenty of septate acute angle dichotomous branching fungal hyphae of Aspergillus species under high power (HE stain, X400).

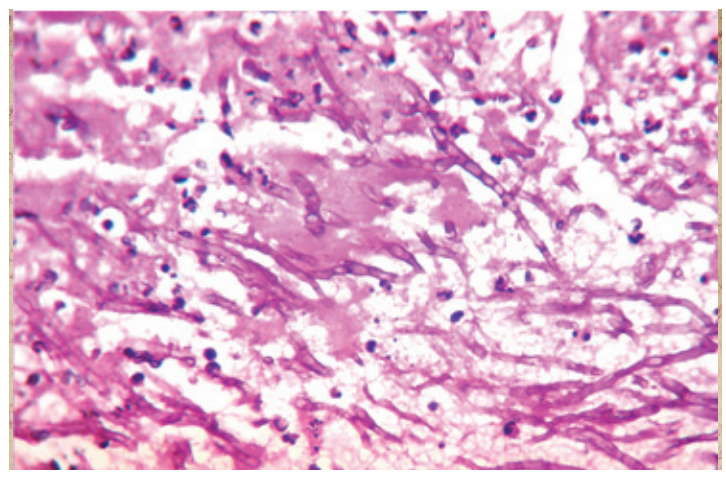

Figure 2: Septate acute angle dichotomous branching fungal hyphae of Aspergillus species by PAS stain (PAS stain, X400).

\section{CONCLUSION}

Our case reinforces the necessity to adopt a combined clinicolaboratory approach for early diagnosis, effective treatment and improved patient survival in cases of endocarditis.

\section{REFERENCES}

1. Boucher HW, Patterson TF. Aspergillosis, In: Hospenthal DR, Rinaldi MG, (eds). Infectious disease: diagnosis and treatment of human mycoses. Humana: New Jersy; 2008;pp181-99. 
2. Kradin RL, Mark EJ. Pulmonary infections. In: Kradin RL, (eds). Diagnostic pathology of infectious disease. Saunders Elservier: Philadelphia; 2010; pp125-88.

3. Kradin RL, Mark EJ. The pathology of pulmonary disorders due to Aspergillus spp. Arch Pathol Lab Med 2008;132:606-14.

4. Atkinson JB, Connor DH, Robinowitz M, McAllister HA, Virmani R Cardiac fungal infections: review of autopsy findings in 60 patients. Hum Pathol 1984;15:935-42.

5. Reyes MP, Lerner AM. Endocarditis caused by Candida species. In: Bodey GP, Fainstein V (eds). Candidiasis. Raven: New York; 1985;pp203-9.
6. Verghese S, Maria CF, Mullaseri AS, Asha M, Padmaja P, Padhye AA. Aspergillus endocarditis presenting as femoral artery embolism. Mycoses 2004;47:252-6.

7. Noordally SO, Sohawon S, De Bels D, Duttmann R, Gottignies P, Devriendt J. Late onset of Aspergillus aortitis presenting as femoral artery embolism following coronary artery bypass graft surgery. Acta Medica 2011;54:175-6.

8. Rana M, Fahad B, Abid Q. Embolic aspergillus endophthalmitis in an immunocompetent patient from aortic root aspergillus endocarditis. Mycoses 2008;51:352-3. 